PROCEEDINGS OF THE

AMERICAN MATHEMATICAL SOCIETY

Volume 138, Number 3, March 2010, Pages 1047-105

S 0002-9939(09)10162-4

Article electronically published on November 10, 2009

\title{
SHADOWING, ENTROPY AND A HOMEOMORPHISM OF THE PSEUDOARC
}

\author{
PIOTR KOŚCIELNIAK AND PIOTR OPROCHA
}

(Communicated by Bryna Kra)

\begin{abstract}
In this article we provide a method of constructing continuous maps $f:[0,1] \rightarrow[0,1]$ such that $f$ is topologically mixing, has the shadowing property, and the inverse limit of copies of $[0,1]$ with $f$ as the bonding map is the pseudoarc. Such a map can be obtained as an arbitrarily small $\mathrm{e}^{0}$-perturbation of any topologically exact map on $[0,1]$. We have therefore answered, in the affirmative, a question posed by Chen and Li in 1993.
\end{abstract}

\section{INTRODUCTION}

The first example of a pseudoarc was obtained by Knaster in [17. Twenty years after Knaster, Moise constructed a nondegenerate chainable hereditarily indecomposable continuum that was similar to an arc in the sense that it was homeomorphic to each of its nondegenerate subcontinua 21. In that paper the name pseudoarc was used for the first time. Later it was proved by Bing that all pseudoarcs are homeomorphic [5], so from the topological point of view the pseudoarc is unique.

While the pseudoarc seems to be a very complicated object, it can appear in dynamics in a natural way. Namely, it can be an invariant set of a dynamical system of the plane. Furthermore, it was proved by Barge that the pseudoarc (or any continua obtained as the inverse limit of an interval map) can be the global attractor in a dynamical system in the plane [2] and, as a consequence, the following natural question arose in the literature:

Question 1. Is there a transitive homeomorphism of the pseudoarc?

This question was answered in 1991 by Kennedy [16] and independently by Minc and Transue 20] in the affirmative. In fact, the construction of Minc and Transue answers another question raised by Barge in 1988 at the Spring Topology Conference (see comment in 20] on p. 1165; some additional bibliographical remarks can be found on p. 640 in [3]).

Question 2. Is there a transitive map $f \in C(I)$ such that the inverse limit of copies of $I$ with $f$ as the bonding map is the pseudoarc?

Received by the editors May 4, 2009, and, in revised form, August 4, 2009.

2000 Mathematics Subject Classification. Primary 37B45; Secondary 54H20, 37B40, 37B05.

Key words and phrases. Pseudoarc, shadowing, entropy, crooked map, topological mixing.

(C)2009 American Mathematical Society Reverts to public domain 28 years from publication 
Recall that the inverse limit of $f \in C(I)$ is the following subset of the Hilbert cube II (the Cartesian product of countably many copies of $I$ ):

$$
\mathbb{X}_{f}=\lim _{\longleftarrow}(I, f)=\left\{\left(x_{0}, x_{1}, \ldots\right): f\left(x_{n+1}\right)=x_{n}\right\} \subset \mathbb{I} .
$$

Note that every map $f \in C(I)$ induces a homeomorphism on the inverse limit $\sigma_{f}: \mathbb{X}_{f} \rightarrow \mathbb{X}_{f}$ defined by $\sigma_{f}\left(x_{0}, x_{1}, \ldots\right)=\left(f\left(x_{0}\right), x_{0}, x_{1}, \ldots\right)$.

While 20] provides a clear way for the construction of transitive maps $f \in C(I)$ whose inverse limit is the pseudoarc, such maps are to some extent very special. Recent results [7, Theorem D] show that the set of surjections whose inverse limit set is the pseudoarc is nowhere dense in the set of all surjections $C_{s}(I) \subset C(I)$ (and obviously, every transitive map $f \in C(I)$ is a surjection). Additionally, if such a map has a periodic point of period 2, then it must have a periodic point of odd period greater than one. But according to Sharkovsky's theorem (see [23] or [8]), if $f \in C(I)$ has a periodic point of period $n \neq 1$, then $f$ has a periodic point of period 2 , so if $\mathbb{X}_{f}$ is the pseudoarc, then the only periodic points of $f$ are fixed points or $f$ has a periodic point with odd period greater than one. It is noteworthy that the question whether there is $f \in C(I)$ such that $\mathbb{X}_{f}$ is the pseudoarc and $f$ has a periodic point of odd period greater than one but without periodic point of period three is still open [19, Question 7.1.8].

The maps constructed according to the scheme of [20] have always a point of odd period greater than one, since transitive maps of the interval always have a point of period 6 [6, Corollary 3.5]. But there are also known examples of maps with $\mathbb{X}_{f}$ being the pseudoarc but without points of period other than 1. A classical example is the map constructed by Henderson in 1964 in [15. This map has 0 and 1 as its only fixed points, and $f(x)<x$ for all $x \in(0,1)$ (its graph looks much like the graph of $g(x)=x^{2}$ with small notches in it). It was proved by Chen and Li that this map has the shadowing property [1], and it is also not hard to verify that the set of nonwandering points of that map equals $\{0,1\}$; in particular this map has zero topological entropy. We do not recall here the definition of topological entropy, since it is well known (e.g. see [14]; for a more detailed exposition in the context of one-dimensional maps, see 11). For our purposes it is only important to remember that topological entropy is a number $h_{\text {top }}(f) \in[0,+\infty]$ and for every transitive map $f \in C(I)$ its topological entropy is at least $h_{\text {top }}(f) \geq \log \sqrt{2}$ (see [6. Corollary 3.6]). It is also known that if $f \in C(I)$, then $h_{\text {top }}(f)=h_{\text {top }}\left(\sigma_{f}\right)$ and if $f$ has the shadowing property, then so does $\sigma_{f}$ (see [10, Proposition 5.2] and [11, Theorem 1.3] respectively). Then it is evident that the map of Henderson induces a homeomorphism $\sigma_{f}$ of the pseudoarc with the shadowing property and zero topological entropy. This observation led Chen and Li to state the following question (see [12, p. 93]), which, so far, hasn't been answered yet.

Question 3. Is there a homeomorphism of the pseudoarc with the shadowing property and positive topological entropy?

The main aim of this article is a construction of a map which provides the positive answer to the above question. To obtain this goal we will modify the construction of Minc and Transue. The main difficulty is that shadowing of $f \in C(I)$, similarly to the property that $\mathbb{X}_{f}$ is the pseudoarc, is not stable under small perturbations. Even in the family of tent maps $f_{s}, s \in[\sqrt{2}, 2]$, the set of parameters for which $f_{s}$ has the shadowing property has full Lebesgue measure, while the complement of 
this set is locally uncountable; that is, its intersection with any open subinterval of $[\sqrt{2}, 2]$ is uncountable [13. Then as we see, the main step is to develop a special class of perturbations, suitable in our context.

\section{BASIC DEFINITIONS AND FACTS}

A continuum is a connected compact space. A chain is a collection of open sets $\left\{C_{i}\right\}_{i=1}^{n}$ (so-called links) such that $C_{i} \cap C_{j} \neq \emptyset$ if and only if $|j-i| \leq 1$. A continuum $X$ is chainable if for all $\varepsilon>0$, there is a chain of open sets of diameters less than $\varepsilon$ that covers $X$. A continuum $X$ is indecomposable if it cannot be expressed as the union of two of its proper subcontinua and hereditarily indecomposable if each of its subcontinua is indecomposable. We say that a nondegenerate continuum is a pseudoarc if it is chainable and hereditarily indecomposable.

In this article we consider continuous maps $f: X \rightarrow X$ acting on a compact metric space $(X, d)$. The space of all such maps $f$ is denoted $C(X)$. We denote $I=[0,1]$ and always assume that $I$ is endowed with the metric $d(x, y)=|x-y|$. We endow $C(I)$ with the complete metric

$$
\rho(f, g)=\sup _{x \in I}|f(x)-g(x)| .
$$

A map $f \in C(X)$ is:

- transitive if for all nonempty open sets $U, V$ there is $n>0$ such that $f^{n}(U) \cap V \neq \emptyset$

- topologically weakly mixing if the Cartesian product $f \times f$ is transitive on $X \times X$,

- topologically mixing if for all nonempty open sets $U, V$ there is $N$ such that $f^{n}(U) \cap V \neq \emptyset$ for every $n>N$,

- topologically exact if for every nonempty open set $U$ there is $n>0$ such that $f^{n}(U)=X$.

A sequence $\left\{y_{n}\right\}_{n \in \mathbb{N}} \subset I$ is called a $\delta$-pseudo-orbit $(\delta \geq 0)$ of $f \in C(I)$ if

$$
d\left(f\left(x_{n}\right), x_{n+1}\right) \leq \delta \text { for every } n \in \mathbb{N} .
$$

We say that a map $f \in C(I)$ has the shadowing property if for every $\varepsilon>0$ there exists $\delta>0$ satisfying the following condition: given a $\delta$-pseudo-orbit $\mathbf{y}=\left\{y_{n}\right\}_{n \in \mathbb{N}}$ we can find a corresponding point $x \in I$ which $\varepsilon$-traces $\mathbf{y}$, i.e.,

$$
d\left(f^{n}(x), y_{n}\right) \leq \varepsilon \text { for every } n \in \mathbb{N} .
$$

Definition 1. Let $f \in C(I)$, let $a, b \in I$ and let $\delta>0$. We say that $f$ is $\delta$-crooked between $a$ and $b$ if, for every two points $c, d \in I$ such that $f(c)=a$ and $f(d)=b$, there is a point $c^{\prime}$ between $c$ and $d$ and there is a point $d^{\prime}$ between $c^{\prime}$ and $d$ such that $\left|b-f\left(c^{\prime}\right)\right|<\delta$ and $\left|a-f\left(d^{\prime}\right)\right|<\delta$. We will say that $f$ is $\delta$-crooked if it is $\delta$-crooked between every pair of points.

We say that $x \in I$ is a critical point of $f \in C(I)$ if $f^{\prime}(x)$ vanishes or is undefined.

Definition 2. A continuous map $f \in C(I)$ is called admissible if it is a piecewise linear and topologically exact map such that $\left|f^{\prime}(x)\right|>4$ for every noncritical point $x \in I$.

Now, we recall some useful facts from [20] and [11] for the reader's convenience. It is recommended, however, to keep a copy of [20] and [1] as a reference when reading the present article. 
Lemma 3 ([20, Proposition 2]). Let $f, F \in C(I)$ be two maps so that $\rho(f, F)<\varepsilon$. If $f$ is $\delta$-crooked, then $F$ is $(\delta+2 \varepsilon)$-crooked.

Lemma 4 ([20, Proposition 3]). Let $\left\{f_{i}\right\}_{i=1}^{\infty} \subset C(I)$ be a sequence of $\delta$-crooked maps. If the sequence converges uniformly, then the limit is also $\delta$-crooked.

Lemma 5 ([20, Lemma on p. 1167]). Let $f \in C(I)$ be an admissible map. Let $\eta$ and $\delta$ be two positive numbers. Then there is an admissible map $F \in C(I)$ and there is a positive integer $n$ such that $F^{n}$ is $\delta$-crooked and $\rho(f, F)<\eta$. Moreover, if $0 \leq a<b \leq 1$ and $b-a \geq \eta$, then $f([a, b]) \subset F([a, b])$ and $F^{n}([a, b])=I$.

Remark 6. It follows from the proof of Lemma 5 (see [20]) that $n$ can be arbitrarily large since $n$ is a positive integer such that $f^{n}([a, b])=I$ for every $0 \leq a<b \leq 1$, $b-a>\varepsilon / 4$, where $\varepsilon>0$ is some particular number fixed during the proof (see the first paragraph on page 1168 in [20]). Namely, if $n$ fulfills the above condition, then so does any integer $m>n$.

Lemma 7 ([20, Proposition 4]). Let $f \in C(I)$ be a map with the property that for every $\delta>0$ there is an integer $n>0$ such that $f^{n}$ is $\delta$-crooked. Then the inverse limit of copies of I with $f$ as the bonding map is the pseudoarc.

Theorem 8 ([11, Theorem 1.3]). If $f \in C(I)$ has the shadowing property, then the homeomorphism $\sigma_{f}$ also has the shadowing property.

\section{Shadowing AND ADMISSIBLE MAPS}

Let $f \in C(I), \lambda>1$ and suppose that the set of critical points of $f$ is nowhere dense in $I$. If $|f(x)-f(y)| \geq \lambda|x-y|$ for every $x, y \in I$ such that $x<y$ and the interval $(x, y)$ contains no critical point, then we say that $f$ is $\lambda$-expanding.

By a nondegenerate interval we mean any closed interval which is not a singleton.

The following fact is well known. We present its proof for completeness.

Lemma 9. Assume that $f \in C(I)$ is $\lambda$-expanding with nowhere dense set of critical points and let $N<\lambda$ be a positive integer. If $J$ is a nondegenerate interval, then there exists an integer $n \geq 0$ such that $f^{n}(J)$ contains at least $N$ distinct critical points.

Proof. Fix any nondegenerate interval $J$ and let $C \subset I$ be the set of critical points of $f$. Note that for any nondegenerate interval $K$ the image $f^{n}(K)$ never reduces to a single point (i.e. it is always a nondegenerate interval), as otherwise int $K \subset C$ for some nondegenerate interval $K$, which contradicts the assumptions.

Suppose that $K$ is a nondegenerate interval that contains no more than $N-1$ critical points. Then $K \backslash C$ has at most $N$ connected components. In particular there are $a<b$ such that $b-a \geq \operatorname{diam} K / N,(a, b) \cap C=\emptyset$ and $[a, b] \subset K$. This implies that

$$
\operatorname{diam} f(K) \geq \operatorname{diam} f([a, b])>\lambda(b-a) \geq(\lambda / N) \operatorname{diam} K .
$$

This immediately implies that if $f^{n}(J)$ contains at most $N-1$ critical points for every $n \geq 0$, then

$$
\operatorname{diam} f^{n}(J) \geq(\lambda / N)^{n} \operatorname{diam} J \longrightarrow+\infty
$$

which is impossible.

Assume that $f \in C(I), f(x)=a$ and $f(y)=b$. We say that $f$ is: 
- linear on $[x, y]$ if $f(z)=a+(z-x) \frac{b-a}{y-x}$.

- two-fold on $[x, y]$ with the peak $c$ if $f\left(\frac{x+y}{2}\right)=c$ and $f$ is linear on intervals $\left[x, \frac{x+y}{2}\right],\left[\frac{x+y}{2}, y\right]$.

- three-fold on $[x, y]$ if $f\left(x+\frac{1}{3}(y-x)\right)=b, f\left(x+\frac{2}{3}(y-x)\right)=a$ and $f$ is linear on each interval $\left[x, x+\frac{1}{3}(y-x)\right],\left[x+\frac{1}{3}(y-x), x+\frac{2}{3}(y-x)\right],\left[x+\frac{2}{3}(y-x), y\right]$.

Theorem 10. For every $\varepsilon>0$ and every topologically exact map $f \in C(I)$ there are $\delta<\frac{\varepsilon}{2}$ and $F \in C(I)$ such that:

(1) $F$ is admissible and $\rho(f, F)<\frac{\varepsilon}{2}$;

(2) if $g \in C(I)$ and $\rho(F, g)<\delta$, then every $\delta$-pseudo-orbit for $g$ is $\varepsilon$-traced.

Proof. Assume that an $\varepsilon>0$ and a topologically exact map $f \in C(I)$ are given. We may assume that $\varepsilon<\frac{1}{2}$. First, we construct a piecewise linear map $F$ and next we will show that it has all the desired properties.

Step 1 (Construction). There exist a sequence $0=a_{0}<a_{1}<\ldots<a_{n}<a_{n+1}=1$ and $0<\gamma<\frac{\varepsilon}{18}$ such that

(i) $a_{i+1}-a_{i}=\gamma$ for $i=0, \ldots, n$;

(ii) $\beta_{i}-\alpha_{i}<\frac{\varepsilon}{3}$ for $i=0,1, \ldots, n$, where

$$
\alpha_{i}=\inf \left\{f(x): x \in\left[a_{i}, a_{i+1}\right]\right\} \quad, \quad \beta_{i}=\sup \left\{f(x): x \in\left[a_{i}, a_{i+1}\right]\right\} .
$$

The map $f$ is topologically exact, so it is not constant on any open interval. In particular $\alpha_{i}<\beta_{i}$.

Put $k_{i}=\max \left\{j: a_{j} \leq \alpha_{i}\right\}$ and $l_{i}=\min \left\{j: a_{j} \geq \beta_{i}\right\}$ and define

$$
\hat{\alpha}_{i}=\left\{\begin{array}{ll}
0, & \text { if } k_{i}=0, \\
\frac{a_{k-1}+a_{k}}{2}, & \text { otherwise, }
\end{array} \quad \hat{\beta}_{i}= \begin{cases}1, & \text { if } l_{i}=n+1, \\
\frac{a_{l}+a_{l+1}}{2}, & \text { otherwise }\end{cases}\right.
$$

We divide each interval $\left[a_{i}, a_{i+1}\right]$ into six subintervals by putting

$$
\begin{gathered}
y_{0}^{i}=a_{i}, \quad y_{6}^{i}=a_{i}+\gamma=a_{i+1}, \quad y_{2}^{i}=a_{i}+\frac{\gamma}{3}, \quad y_{3}^{i}=a_{i}+\frac{\gamma}{2}, \quad y_{4}^{i}=a_{i}+\frac{2 \gamma}{3}, \\
y_{1}^{i}=a_{i}+\frac{\gamma}{8}, \quad y_{5}^{i}=a_{i}+\frac{7 \gamma}{8}
\end{gathered}
$$

We define $b_{0}^{i}=f\left(a_{i}\right), b_{6}^{i}=f\left(a_{i+1}\right), b_{2}^{i}=b_{3}^{i}=b_{4}^{i}=\hat{\alpha}_{i}$. We also put

$$
b_{1}^{i}=\left\{\begin{array}{ll}
\hat{\alpha}_{i}, & b_{0}^{i}-\hat{\alpha}_{i}>\frac{\gamma}{2}, \\
\hat{\beta}_{i}, & \text { otherwise },
\end{array} \quad b_{5}^{i}= \begin{cases}\hat{\alpha}_{i}, & b_{6}^{i}-\hat{\alpha}_{i}>\frac{\gamma}{2}, \\
\hat{\beta}_{i}, & \text { otherwise }\end{cases}\right.
$$

Now we put $F\left(y_{j}^{i}\right)=b_{j}^{i}, j=0, \ldots, 6$ and extend the definition onto $\left[a_{i}, a_{i+1}\right]$ in the following way:

(i) $F$ is linear on $\left[y_{0}^{i}, y_{1}^{i}\right]$ and $\left[y_{5}^{i}, y_{6}^{i}\right]$.

(ii) $F$ is two-fold on both intervals $\left[y_{2}^{i}, y_{3}^{i}\right],\left[y_{3}^{i}, y_{4}^{i}\right]$ with the peak $\hat{\beta}_{i}$.

(iii) On $\left[y_{1}^{i}, y_{2}^{i}\right]$ the map $F$ is two-fold with the peak $\hat{\beta}_{i}$ or three-fold when the values on endpoints are equal or different respectively (see the definition of two-fold and three-fold maps given just before the theorem). On $\left[y_{4}^{i}, y_{5}^{i}\right]$ the map $F$ is defined the same way.

Step 2 (Admissibility). Now, we will show that $\rho(f, F)<\frac{\varepsilon}{2}$ and $F$ is admissible. Obviously $F$ is piecewise linear. The remaining conditions are as follows: 
Claim 2.1. $\rho(f, F)<\frac{\varepsilon}{2}$. Observe that

$$
\begin{aligned}
\hat{\beta}_{i}-\hat{\alpha}_{i} & \leq \hat{\beta}_{i}-\beta_{i}+\beta_{i}-\alpha_{i}+\alpha_{i}-\hat{\alpha}_{i} \\
& \leq \frac{3}{2} \gamma+\frac{\varepsilon}{3}+\frac{3}{2} \gamma \\
& <\frac{\varepsilon}{12}+\frac{\varepsilon}{3}+\frac{\varepsilon}{12}=\frac{\varepsilon}{2} .
\end{aligned}
$$

By the definition of $F$ we obtain that

$$
f\left(\left[a_{i}, a_{i+1}\right]\right)=\left[\alpha_{i}, \beta_{i}\right] \subset\left[\hat{\alpha}_{i}, \hat{\beta}_{i}\right]=F\left(\left[a_{i}, a_{i+1}\right]\right)
$$

and so $|f(x)-F(x)|<\frac{\varepsilon}{2}$ for every $x \in\left[a_{i}, a_{i+1}\right]$. Since $i$ was arbitrary, the claim holds.

Claim 2.2. $\left|F^{\prime}(t)\right|>4$ wherever it exists. Fix $i \in\{0, \ldots, n\}$. By the definition, $\hat{\beta}_{i}-\hat{\alpha}_{i} \geq \frac{3 \gamma}{2},\left|b_{1}^{i}-b_{0}^{i}\right|<\frac{\gamma}{2}$ and $\left|b_{6}^{i}-b_{5}^{i}\right|<\frac{\gamma}{2}$. Then for any noncritical $t \in\left[y_{0}^{i}, y_{1}^{i}\right]$ or $t \in\left[y_{5}^{i}, y_{6}^{i}\right]$ we have that $\left|F^{\prime}(t)\right|>\frac{\gamma}{2} \frac{8}{\gamma}=4$. The map $F$ is at least twofold on each interval $\left[y_{j}^{i}, y_{j+1}^{i}\right]$ and $y_{j+1}^{i}-y_{j}^{i}<\frac{\gamma}{3}$. This immediately implies that $\left|F^{\prime}(t)\right| \geq \frac{3 \gamma}{2} \frac{6}{\gamma}>4$ for each noncritical argument $t \in\left[y_{j}^{i}, y_{j+1}^{i}\right]$.

Claim 2.3. $f^{k}\left(\left[a_{i}, a_{i+1}\right]\right) \subset F^{k}\left(\left[a_{i}, a_{i+1}\right]\right)$ for every $k>0$ and every $i=0, \ldots, n$ and the following implication holds:

$$
\text { if } \begin{aligned}
& F^{k}\left(\left[a_{i}, a_{i+1}\right]\right) \cap\left(a_{j}, a_{j+1}\right) \neq \emptyset \text {, then } \\
& F^{k}\left(\left[a_{i}, a_{i+1}\right]\right) \supset\left[a_{j}, \frac{a_{j}+a_{j+1}}{2}\right] \text { or } F^{k}\left(\left[a_{i}, a_{i+1}\right]\right) \supset\left[\frac{a_{j}+a_{j+1}}{2}, a_{j+1}\right] .
\end{aligned}
$$

We will prove the claim by induction on $k$. For $k=1$ the first part of the claim holds by (3.1), and condition (3.2) holds by the definition of $\hat{\alpha}_{i}$ and $\hat{\beta}_{i}$. Next, fix $k>0$ and assume that the claim holds for $i=0, \ldots, n$ and all $s \leq k$. Fix an integer $i \in\{0, \ldots, n\}$. The set $F^{k}\left(\left[a_{i}, a_{i+1}\right]\right)$ is closed and connected; thus it is a closed interval, say $F^{k}\left(\left[a_{i}, a_{i+1}\right]\right)=[a, b]$. Let $\hat{i}=\max \left\{j: a_{j} \leq a\right\}, \hat{j}=$ $\min \left\{j: a_{j} \geq b\right\}$. Note that $b-a \geq \frac{3}{2} \gamma$ so $\hat{j}-\hat{i} \geq 2$. By condition (3.2) we obtain that

$$
\left[\frac{a_{\hat{i}}+a_{\hat{i}+1}}{2}, \frac{a_{\hat{j}-1}+a_{\hat{j}}}{2}\right] \subset[a, b] .
$$

Note that by the definition of $F$ the following condition holds for $j=0, \ldots, n$ :

$$
F\left(\left[a_{j}, \frac{a_{j}+a_{j+1}}{2}\right]\right)=F\left(\left[\frac{a_{j}+a_{j+1}}{2}, a_{j+1}\right]\right)=F\left(\left[a_{j}, a_{j+1}\right]\right) .
$$

Combining (3.3) and (3.4) we get

$$
F^{k+1}\left(\left[a_{i}, a_{i+1}\right]\right)=F([a, b])=\bigcup_{j=\hat{i}}^{\hat{j}-1} F\left(\left[a_{j}, a_{j+1}\right]\right),
$$

and so the proof of the claim is finished by the assumptions, since $k \geq 1$.

Claim 2.4. $F$ is topologically exact. By the construction, $F$ is $\lambda$-expanding with some $\lambda>4$. Fix any nondegenerate interval $J$. By Lemma 9 there is $k>0$ such that $F^{k}(J)$ contains at least four critical points. Then there are $i \in\{0, \ldots, n\}$ and $j \in\{1, \ldots, 4\}$ such that $F^{k}(J) \supset[c, d]$, where $c<d$ are two consecutive critical points in $\left[y_{j}^{i}, y_{j+1}^{i}\right]$. But then there is $s$ such that $F^{k+1}(J) \supset\left[a_{s}, a_{s+1}\right]$.

By Claim 2.3 we see that $F^{k+j+1}(J) \supset f^{j}\left(\left[a_{s}, a_{s+1}\right]\right)$ for every $j \geq 0$. In particular there is $m$ such that $F^{m}(J)=I$, since $f$ is topologically exact. 
Step 3 ( $\varepsilon$-shadowing). Put $\delta=\frac{\gamma}{10}$. Take any $g \in C(I)$ such that $\rho(F, g)<\delta$ and let $\mathbf{x}=\left\{x_{i}\right\}_{i=0}^{\infty}$ be a $\delta$-pseudo-orbit for $g$. We claim that there is a sequence of intervals $J_{i}$ such that

(1) $\operatorname{diam} J_{i} \leq \gamma$ and if $i>0$, then $J_{i} \subset g\left(J_{i-1}\right)$;

(2) $\operatorname{dist}\left(x_{i}, J_{i}\right)<\gamma$.

(3) for every $i$ there is $p$ such that $F\left(J_{i}\right)=F\left(\left[a_{p}, a_{p+1}\right]\right)$ and $x_{i} \in\left[a_{p}, a_{p+1}\right]$.

Take $p$ such that $\left[a_{p}, a_{p+1}\right] \ni x_{0}$ and put $J_{0}=\left[a_{p}, a_{p+1}\right]$. Then conditions (11)-(3) are fulfilled for $i=0$.

Next assume that for $i=0, \ldots, m$ there are intervals $J_{i}$ such that conditions (11) -(3) are satisfied. We will show how to construct $J_{m+1}$. Denote $F\left(J_{m}\right)=[a, b]$. By (3) and arguments similar to (3.3) we obtain integers $\hat{i}, \hat{j}, \hat{j}-\hat{i} \geq 2$ such that

$$
\left[\frac{a_{\hat{i}}+a_{\hat{i}+1}}{2}, \frac{a_{\hat{j}-1}+a_{\hat{j}}}{2}\right] \subset[a, b]
$$

and by the definition of $F$ we have $a=0$ or $a=\frac{a_{\hat{\imath}}+a_{\hat{\imath}+1}}{2}, b=1$ or $b=\frac{a_{\hat{j}-1}+a_{\hat{j}}}{2}$. Then

$$
g\left(J_{m}\right) \supset[a+\delta, b-\delta] \supset\left[a_{\hat{i}+1}-\frac{\gamma}{3}, a_{\hat{j}-1}+\frac{\gamma}{3}\right]
$$

and $g\left(x_{m}\right) \in[a-\delta, b+\delta]$, which implies that

$$
x_{m+1} \in[a-2 \delta, b+2 \delta] \subset\left[a_{\hat{i}}, a_{\hat{j}}\right] .
$$

Then there is $\hat{i} \leq q \leq \hat{j}$ such that $x_{m+1} \in\left[a_{q}, a_{q+1}\right]$ and $L \subset\left[a_{\hat{i}+1}-\frac{\gamma}{3}, a_{\hat{j}-1}+\frac{\gamma}{3}\right]$ or $R \subset\left[a_{\hat{i}+1}-\frac{\gamma}{3}, a_{\hat{j}-1}+\frac{\gamma}{3}\right]$, where $L=\left[a_{q}, a_{q}+\frac{\gamma}{3}\right]$ and $R=\left[a_{q+1}-\frac{\gamma}{3}, a_{q+1}\right]$. Now, we put $J_{m+1}=L$ or $J_{m+1}=R$ depending on the situation, obtaining that $J_{m+1} \subset g\left(J_{m}\right)$. Additionally $\operatorname{dist}\left(x_{m+1}, J_{m+1}\right)<\gamma$ and by the definition of $F$ it follows that

$$
F\left(J_{m+1}\right)=F(L)=F(R)=F\left(\left[a_{q}, a_{q+1}\right]\right) .
$$

The inductive construction is finished.

By (11) there is a point $z \in I$ such that $z \in \bigcap_{i=0}^{\infty} g^{-i}\left(J_{i}\right)$. Then $g^{i}(z) \in J_{i}$ for every $i \geq 0$ and so by (11) and (2) we obtain that

$$
d\left(g^{i}(z), x_{i}\right) \leq \operatorname{diam} J_{i}+\operatorname{dist}\left(x_{i}, J_{i}\right) \leq 2 \gamma<\varepsilon .
$$

We have just proved that the pseudo-orbit $\mathbf{x}$ is $\varepsilon$-traced by the point $z$.

Theorem 11. For every topologically exact map $T \in C(I)$ and every $\tau>0$ there is a topologically mixing map $f \in C(I)$ with shadowing, such that $\rho(T, f)<\tau$ and the inverse limit of copies of $I$ with $f$ as the bonding map is the pseudoarc.

Proof. First, we will construct a sequence of admissible maps $F_{1}, F_{2}, \ldots$, an increasing sequence of positive integers $N(1)<N(2)<\ldots$, and two sequences of positive numbers $\varepsilon_{1}, \varepsilon_{2}, \ldots, \delta_{1}, \delta_{2}, \ldots$ with the following properties:

(a) $\rho\left(F_{i+1}, F_{i}\right)<\delta_{i}<2^{-i}$ for all $i, \delta_{1}<\tau / 2$;

(b) $F_{i}^{N(k)}$ is $a_{i}\left(2^{-k}-2^{-k-i}\right)$-crooked for all $1 \leq k \leq i$, where

$$
a_{i}=\sum_{j=1}^{i} \frac{1}{j^{2}} ;
$$

(c) there is $\xi>0$ such that if $b-a \geq 2^{-k}$, then $F_{i}^{N(k)}([a, b]) \supset\left[\frac{1}{k}-\xi, 1+\xi-\frac{1}{k}\right]$ for all $1 \leq k \leq i$;

(d) for all $i$, if $\rho\left(F_{i}, g\right)<2 \delta_{i}$, then every $\delta_{i}$-pseudo-orbit for $g$ is $\varepsilon_{i}$-traced; 
(e) $\delta_{i+1}<\delta_{i} / 2$ and $\varepsilon_{i+1}<\varepsilon_{i} / 2$.

Put $\varepsilon_{1}=\varepsilon=\min \{1 / 2, \tau / 4\}$ and apply Theorem [10 to the map $T$ obtaining a number $\nu>0$ and an admissible map $G \in C(I), \rho(G, T)<\frac{\tau}{8}$ such that if $g \in C(I)$ and $\rho(G, g)<\nu$, then every $\nu$-pseudo-orbit of $g$ is $\varepsilon$ traced. Put $a_{1}=$ 1 and $\delta_{1}=\min \{\nu / 4, \tau / 4\}$. Now apply Lemma 5 with $\eta=\min \left\{\delta_{1}, 2^{-1}\right\}$ and $\delta=a_{1}\left(2^{-1}-2^{-1-1}\right)=1 / 4$ obtaining an admissible map $F_{1}$ and $N(1)$ such that (b) and (c) are fulfilled. Additionally, if $\rho\left(F_{1}, g\right)<2 \delta_{1}$ for some $g \in C(I)$, then $\rho(G, g)<3 \delta_{1}<\nu$, which gives $(\mathrm{d})$. Note that $\rho\left(F_{1}, T\right)<\tau / 2$.

Now let us assume that we have already constructed $F_{1}, \ldots, F_{i-1}, N(1), \ldots$, $N(i-1), \varepsilon_{1}, \ldots, \varepsilon_{i-1}$ and $\delta_{1}, \ldots, \delta_{i-1}$.

There exists $0<\eta_{1}<\min \left\{\delta_{i-1}, 2^{-i}\right\}$ with the property that for any $g \in C(I)$ if $\rho\left(F_{i-1}, g\right)<\eta_{1}$, then $\rho\left(F_{i-1}^{N(k)}, g^{N(k)}\right)<a_{i-1} 2^{-k-i-1}$ for all $k<i$. In particular, it follows by Lemma 3 that $g^{N(k)}$ is $a_{i-1}\left(2^{-k}-2^{-k-i}\right)$-crooked. By (c), there is $\mu$ such that if $b-a \geq 2^{-k}$, then $F_{i-1}^{N(k)}([a, b]) \supset\left[\frac{1}{k}-\mu, 1+\mu-\frac{1}{k}\right]$ for all $1 \leq k<i$. Put $\xi=\mu / 2$ and decrease $\eta_{1}$ if necessary, so that $g^{N(k)}([a, b]) \supset\left[\frac{1}{k}-\mu, 1+\mu-\frac{1}{k}\right]$ for every $b-a \geq 2^{-k}, 1 \leq k<i$ and $g \in C(I)$ with $\rho\left(F_{i-1}, g\right)<\eta_{1}$. The constant $\eta_{1}$ will be essential in our construction.

Apply Theorem [10 with $\varepsilon=\varepsilon_{i}=\min \left\{\frac{\eta_{1}}{4}, \frac{\varepsilon_{i-1}}{4}\right\}$ and $f=F_{i-1}$, obtaining an admissible map $G \in C(I)$ and $\gamma>0$ such that if $g \in C(I)$ and $\rho(g, G)<\gamma$, then every $\gamma$-pseudo-orbit of $g$ is $\varepsilon_{i}$-traced. Additionally $\rho\left(F_{i-1}, G\right)<\frac{\eta_{1}}{4}$ and so by the definition of $\eta_{1}$ the map $G^{N(k)}$ is $a_{i-1}\left(2^{-k}-2^{-k-i}\right)$-crooked for all $k<i$. Put $\delta_{i}=\min \left\{\frac{\gamma}{4}, \frac{\delta_{i-1}}{4}\right\}$ and note that $\delta_{i}<2^{-i}$ and (e) holds.

There is $\eta_{2}>0$ such that for all $g \in C(I)$, if $\rho(g, G)<\eta_{2}$, then

$$
\rho\left(g^{N(k)}, G^{N(k)}\right)<\left(1 /\left(2 i^{2}\right)\right)\left(2^{-k}-2^{-k-i}\right)
$$

for all $k<i$. Now, let us use Lemma 5 (together with Remark 6) for $f=G$, $\eta=\min \left\{\frac{\eta_{1}}{2}, \frac{\eta}{2}, 2^{-i-1}, \delta_{i}\right\}$ and $\delta=a_{i}\left(2^{-i}-2^{-i-i}\right)$ obtaining an admissible map $F_{i} \in C(I)$ and $n>N(i-1)$ such that $\rho\left(F_{i}, G\right)<\eta$ and $F_{i}^{n}$ is $a_{i}\left(2^{-i}-2^{-i-i}\right)$ crooked. Put $N(i)=n$. Note that

$$
\rho\left(F_{i}, F_{i-1}\right)<\eta+\frac{\eta_{1}}{4}<\eta_{1}<\delta_{i-1},
$$

which gives (a). By the definition of $\eta_{1}$ together with the choice of $N(i)$ we obtain (b). Condition (c) is a consequence of the choice of $N(i)$ (Lemma 5) and $\eta_{1}$ $\left(\rho\left(F_{i}, F_{i-1}\right)<\eta_{1}\right)$. Finally, if $g \in C(I)$ and $\rho\left(g, F_{i}\right)<2 \delta_{i}$, then $\rho(G, g)<3 \delta_{i}<\gamma$, which gives $(\mathrm{d})$.

By the induction, a sequence of maps fulfilling (a)-(e) is constructed.

From the properties (a) and (e) the sequence $F_{1}, F_{2}, \ldots$ converges to a map $f \in C(f)$ and additionally

$$
\rho\left(f, F_{1}\right)<\sum_{i=1}^{\infty} \delta_{i} \leq \sum_{i=1}^{\infty} 2^{-i+1} \delta_{1} \leq 2 \delta_{1} \leq \frac{\tau}{2} .
$$

If $U \subset I$ is a nonempty open set, then there are $a<b$ and $N>0$ such that $[a, b] \subset U$ and $b-a \geq 2^{-k}$ for every $k \geq N$. But then $f^{N(k)}(U) \supset\left[\frac{1}{k}, 1-\frac{1}{k}\right]$, which shows that $f$ is topologically weakly mixing (e.g. see [18, Lemma 24]). But it is well known that in the context of interval maps, weak mixing implies mixing (e.g. it can be easily deduced from [4, Theorem 6]). Put $M=\sum_{j=1}^{\infty}\left(1 / j^{2}\right)$ and 
observe that the property (b) and Lemma 4 imply that $f^{N(k)}$ is $2^{-k} M$-crooked for all $k=1,2, \ldots$. By Lemma 7 we obtain that the inverse limit of copies of $I$ with $f$ as the bonding map is the pseudoarc.

Finally, we prove that $F$ has the shadowing property. Fix any $\varepsilon>0$. Then there is $i$ such that $\varepsilon_{i}<\varepsilon$. By (d) and (e) we obtain that $\rho\left(f, F_{i}\right)<\delta_{i}$ and obviously each $\delta_{i}$-pseudo-orbit of $f$ is $\varepsilon$-traced. Indeed, $f$ has the shadowing property.

To finish the proof, note that

$$
\begin{aligned}
\rho(T, f) & \leq \rho\left(T, F_{1}\right)+\rho\left(F_{1}, f\right) \\
& <\frac{\tau}{2}+\frac{\tau}{2}=\tau .
\end{aligned}
$$

Recall that the endpoint $a \in\{0,1\}$ is accessible for a map $f \in C(I)$ if there is $x \in(0,1)$ and $n>0$ such that $f^{n}(x)=a$. A map $f \in C(I)$ is topologically exact if and only if it is topologically mixing and both endpoints $\{0,1\}$ are accessible for $f$ (see [9, Lemma 8.5]; the proof is very simple and can be easily deduced from [18, Lemma 23]).

Remark 12. The maps $f \in C(I)$ constructed in 20] are topologically exact, while the maps provided by Theorem 10 are only topologically mixing. The main difference is that in our case it may happen that one of the endpoints is not accessible.

Corollary 13. There is a topologically exact map $f \in C(I)$ with the shadowing property such that the inverse limit of copies of I with $f$ as the bonding map is the pseudoarc. In particular $h_{\text {top }}(f) \geq \log \sqrt{2}>0$.

Proof. Let $T \in C(f)$ be the standard tent map; that is, $T(0)=T(1)=0$ and $T$ is two-fold on $[0,1]$ with the peak 1 . Before we proceed further with the proof, let us make two important observations.

(1) Note that the perturbation of any map obtained by Theorem 10 does not change the image of any of the endpoints.

(2) The perturbation $F$ of a map $f \in C(I)$ in Lemma 5 is obtained as the composition $F=f \circ g$ (see the second paragraph on p. 1168 in [20]), where the map $g$ is the map provided by [20, Proposition 5] (the formula for that map depends on the range of perturbation defined in terms of $\varepsilon$ and $\gamma$ ). But the map $g$ provided by [20, Proposition 5] has always (i.e. for any value of $\varepsilon$ and $\gamma$ ) the property that $g(0)=0$ and $g(1)=1$. In particular $F(0)=f(0)$ and $F(1)=f(1)$, which means that this perturbation does not change the image of the endpoints.

By the above remarks, every map $F_{i}$ defined during the proof of Theorem 11 (in the particular case of the tent map $T$ ) has the property that $F_{i}(0)=T(0)=0$ and $F_{i}(1)=T(1)=0$. The same property transfers to the map $f \in C(I)$ in the statement of Theorem 11] which is obtained as the limit of the sequence $F_{i}$ in $(C(I), \rho)$. This means that both endpoints are accessible for $f$, because $f$ is a surjective map and $f(\{0,1\})=\{0\}$. As a consequence, the map $f$ is topologically exact, since it is a topologically mixing map with accessible endpoints.

To finish the proof note that $h_{t o p}(f) \geq \log \sqrt{2}>0$, since $f$ is transitive.

Remark 14. Even if $f$ is topologically exact, $\sigma_{f}$ is only topologically mixing; namely, homeomorphisms can never be topologically exact. 
The map from Corollary 13 defines the homeomorphism $\sigma_{f}$ with interesting dynamical properties. Strictly speaking, by results of [12], we have the following:

Corollary 15. There is a topologically mixing homeomorphism $\xi$ of the pseudoarc with the shadowing property and positive topological entropy $\left(h_{t o p}(\xi) \geq \log \sqrt{2}\right)$.

Proof. It is enough to consider a topologically mixing map $f \in C(I)$ with shadowing and such that $\mathbb{X}_{f}$ is the pseudoarc (e.g. that provided by Corollary 13) to obtain that the homeomorphism $\sigma_{f}: \mathbb{X}_{f} \rightarrow \mathbb{X}_{f}$ has all the claimed properties.

Remark 16. Recently, it was announced by Chris Mouron (at the Spring Topology and Dynamics Conference 2009, Gainesville, FL, USA) that when $f \in C(I)$ and $\mathbb{X}_{f}$ is the pseudoarc, then the topological entropy of $\sigma_{f}$ (hence also of $f$ ) is either 0 or infinity 22 .

\section{ACKNOWLEDGEMENTS}

The research of the second author leading to results included in the paper has received funding from the European Community's Seventh Framework Programme (FP7/2007-2013) under grant agreement No. 219212, belonging to a Marie Curie Intra-European Fellowship for Career Development. The second author was also supported by the Polish Ministry of Science and Higher Education grant No. NN201272333 for years 2007-2009 and AGH local grant No. 10.420.03. The financial support of these institutions is hereby gratefully acknowledged.

\section{REFERENCES}

[1] L. Alsedà, J. Llibre, and M. Misiurewicz, Combinatorial dynamics and entropy in dimension one, second ed., Advanced Series in Nonlinear Dynamics, vol. 5, World Scientific Publishing Co. Inc., River Edge, NJ, 2000. MR1807264 (2001j:37073)

[2] M. Barge, A method for constructing attractors, Ergodic Theory Dynam. Systems 8 (1988), 331-349. MR961734 (90a:58100)

[3] M. Barge and J. Kennedy, Continuum theory and topological dynamics, Open problems in topology, North-Holland, Amsterdam, 1990, pp. 633-644. MR1078669

[4] M. Barge and J. Martin, Dense periodicity on the interval, Proc. Amer. Math. Soc. 94 (1985), 731-735. MR792293 (87b:58068)

[5] R. H. Bing, Concerning hereditarily indecomposable continua, Pacific J. Math. 1 (1951), 43-51. MR0043451 (13:265b)

[6] L. Block and E. M. Coven, Topological conjugacy and transitivity for a class of piecewise monotone maps of the interval, Trans. Amer. Math. Soc. 300 (1987), 297-306. MR871677 (88c:58032)

[7] L. Block, J. Keesling, and V. V. Uspenskij, Inverse limits which are the pseudoarc, Houston J. Math. 26 (2000), 629-638. MR1823960 (2002b:54040)

[8] L. S. Block and W. A. Coppel, Dynamics in one dimension, Lecture Notes in Mathematics, vol. 1513, Springer-Verlag, Berlin, 1992. MR,1176513 (93g:58091)

[9] A. M. Blokh, The "spectral" decomposition for one-dimensional maps, Dynamics reported, Dynam. Report. Expositions Dynam. Systems (N.S.), vol. 4, Springer, Berlin, 1995, pp. 1-59. MR.1346496 (96e:58087)

[10] R. Bowen, Topological entropy and axiom A, Global Analysis (Proc. Sympos. Pure Math., Vol. XIV, Berkeley, Calif., 1968), Amer. Math. Soc., Providence, RI, 1970, pp. 23-41. MR0262459(41:7066)

[11] L. Chen and S. H. Li, Shadowing property for inverse limit spaces, Proc. Amer. Math. Soc. 115 (1992), 573-580. MR.1097338 (92i:58094)

[12] L. Chen and S. H. Li, Dynamical connections between a continuous map and its inverse limit space, Continuum theory and dynamical systems, Lecture Notes in Pure and Appl. Math., vol. 149, Dekker, New York, 1993, pp. 89-97. MR1235348(94d:54089) 
[13] E. M. Coven, I. Kan, and J. A. Yorke, Pseudo-orbit shadowing in the family of tent maps, Trans. Amer. Math. Soc. 308 (1988), 227-241. MR.946440 (90b:58236)

[14] M. Denker, C. Grillenberger, and K. Sigmund, Ergodic theory on compact spaces, Lecture Notes in Mathematics, Vol. 527, Springer-Verlag, Berlin, 1976. MR0457675 (56:15879)

[15] G. W. Henderson, The pseudo-arc as an inverse limit with one binding map, Duke Math. J. 31 (1964), 421-425. MR0166766 (29:4039)

[16] J. Kennedy, A transitive homeomorphism on the pseudoarc which is semiconjugate to the tent map, Trans. Amer. Math. Soc. 326 (1991), 773-793. MR.1010412 (91k:54062)

[17] B. Knaster, Un continu dont tout sous-continu est indécomposable, Fund. Math. 3 (1922), $247-286$.

[18] D. Kwietniak and P. Oprocha, Topological entropy and chaos for maps induced on hyperspaces, Chaos Solitons Fractals 33 (2007), no. 1, 76-86. MR2301847 (2008b:37024)

[19] S. Macías, Topics on continua, Chapman \& Hall/CRC, Boca Raton, FL, 2005. MR2147759 (2006f:54035)

[20] P. Minc and W. R. R. Transue, A transitive map on $[0,1]$ whose inverse limit is the pseudoarc, Proc. Amer. Math. Soc. 111 (1991), 1165-1170. MR.1042271 (91g:54050)

[21] E. E. Moise, An indecomposable plane continuum which is homeomorphic to each of its nondegenerate subcontinua, Trans. Amer. Math. Soc. 63 (1948), 581-594. MR0025733 (10:56i)

[22] C. Mouron, Entropy of shift maps of the pseudo-arc, preprint.

[23] O. M. Šarkovs'kiū, Co-existence of cycles of a continuous mapping of the line into itself, Ukrain. Mat. Z. 16 (1964), 61-71. MR0159905(28:3121)

Institute of Mathematics of the Jagiellonian University, ul. Lojasiewicza 6, 30-348 Kraków, POLAND

E-mail address: piotr.koscielniak@im.uj.edu.pl

Departamento de Matemáticas, Universidad de Murcia, Campus de Espinardo, 30100 Murcia, Spain - And - Faculty of Applied Mathematics, AGH University of Science and Technology, al. Mickiewicza 30, 30-059 Kraków, Poland

E-mail address: oprocha@agh.edu.pl 